\title{
On the MAC protocols for Radio over Fiber indoor networks
}

\author{
Bao Linh Dang, R. Venkatesha Prasad, Ignas Niemegeers \\ Electrical Engineering, Mathematics, Computer Science Faculty \\ Delft University of Technology \\ Mekelweg 4, 2628 CD, Delft, Netherlands \\ Email: \{1.baodang, vprasad,i.g.m.m.niemegeers\}@ewi.tudelft.nl
}

\begin{abstract}
Radio over Fiber (RoF) techniques have been long recognized as the promising solution for indoor networking at millimeter-wave bands. We discuss the possible deployment scenarios of an RoF network for indoor environment. In this paper, we try to find the answer to the question as to which Medium Access Control (MAC) protocol is suitable for an RoF network. To substantiate our claims, performance analysis of two popular MAC protocols employing RoF - IEEE 802.11 representing the distributed control protocol family and ETSI HiperLAN/2 representing the centralized family - is presented. We show that RoF techniques can be applied to both protocols. We also show that the centralized control protocol is an appropriate candidate for RoF networks. This study is expected to contribute to the speedy deployment of RoF for indoor network deployment.
\end{abstract}

\section{INTRODUCTION}

The world of broadband wireless communications is now making inroads into every household. Two aspects are driving this penetration - the booming of wireless technologies that are able to substitute the wired counterparts and the proliferation of entertaining multimedia applications. On the way to realize the future multimedia wireless network, one of the key challenges has always been the limited bandwidth that constrains the maximum datarate of a wireless network. In fact the bandwidth requirement has never been more critical since a number of new multimedia services, such as HDTV or remote IPTV set-top boxes etc., require datarate of up to several hundreds of Mbps.

Moreover, as the users demand for better services increases rapidly, next to the networks capacity required for actual applications, there is much additional capacity needed for QoS provisioning, dynamic resource allocations, security protocols for data integrity etc. In [1], the author has motivated that the bandwidth scarcity can be addressed by utilizing the millimeter wave bands, e.g., the $17 \mathrm{GHz}$ and $60 \mathrm{GHz}$ bands, where abundant spectrum is available. Indoor networks operating at these bands will comprise a number of picocells. Each picocell served by an Access Point (AP) can span only a single room since radio signals are not able to penetrate walls at such high frequencies. Thus a large number of APs are required to cover an indoor space making the cost of the infrastructure drastically increase. The task of simplifying the complexity of APs is therefore becoming increasingly important.

To address the problems of signal propagation at millimeter wave bands and simplifying the complexity of APs, Radio over
Fiber $(\mathrm{RoF})$ is one of the most promising solutions [2]. By integrating the wireless and wired networks, the capacity and transparency of optical networks can be combined with the flexibility and mobility of wireless access networks to form a seamless and scalable communication network. Moreover, this combination enables the concepts of in-house millimeterwave networks where each room is a separated picocell. By using such an architecture, a large part of the complexity of a traditional AP can be transferred to a central control point.

The architecture of a RoF network operating at a millimeter wave band is illustrated in Fig. 1. The RoF network comprises three main components, i.e., simplified APs which are essentially simple antennas (AT_i), an optical distribution network and a central processing unit - the Residential Gateway (RG). To guarantee good signal coverage for the whole indoor area, an antenna is required in each room. All the antennas are connected to the RG via the optical distribution network. Under this approach, the optical distribution system will act as an analogue transmission system that is completely transparent to the radio signals flowing in the networks.

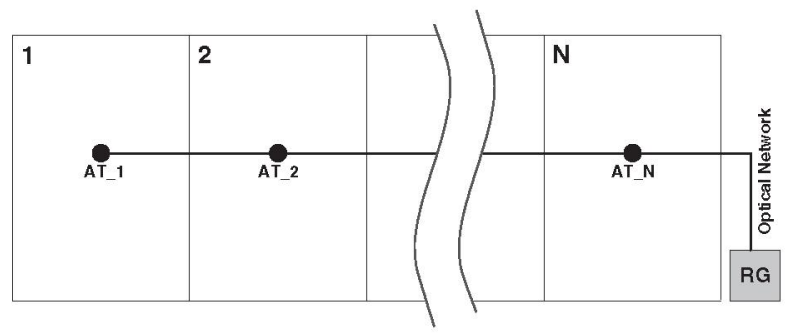

Fig. 1. Indoor networks employing Radio over Fiber

In this paper, we present an analytical study in order to find a suitable Medium Access Control (MAC) protocol for RoF networks. A major effect when an optical distribution system is inserted in a traditional wireless network is the additional propagation delay introduced by the fiber links. This additional propagation delay can exceed the timing boundary of the network's MAC protocol and eventually stop the network from working. For centralized MAC schemes, such as HiperLAN/2 and IEEE 802.16, the effect of the additional propagation delay is less severe since the timing between different phases are allowed to be adjusted by the Base Station (BS). However, 
for distributed control protocols, such as IEEE 802.11 and IEEE 802.15.3, the additional delay poses a challenge to the design of the fiber distribution system. Nevertheless, we discuss that $\mathrm{RoF}$ techniques can be applied to both centralized and distributed control protocols.

As mentioned above, at millimeter wave bands, a radio cell is typically confined in a room. Consequently, MSs in a radio cell will be completely hidden to other MSs in other rooms. This hidden terminal problem is especially severe in distributed control networks where all MSs contend for the channel. In this environment, the well-known RTS/CTS mechanism of IEEE 802.11 is not effective since the RTS and CTS packets will be received by MSs in different rooms. This paper also provides a simple analytical approach that is able to quantify the effects of this hidden-terminal problem on the performance of a IEEE 802.11 based RoF system.

The rest of the paper is organized as follows. In the next section, the fundamental features of the two popular WLAN protocols, namely IEEE 802.11 and ETSI HiperLAN/2, will be revisited. A performance study of IEEE 802.11 and HiperLAN/2 networks employing RoF is presented in Section III along with some analytical results. Finally, we conclude in Section IV.

\section{STATE-OF-THE-ART WIRELESS STANDARDS FOR INDOOR NETWORKING}

In this section, the basic features of the two MAC protocols, namely IEEE 802.11 and ETSI HiperLAN/2, will be presented. As introduced in Section I, IEEE 802.11 MAC protocol based on the Carrier Sense Multiple Access with Collision Avoidance (CSMA/CA) mechanism represents the distributed control protocol family. Meanwhile, HiperLAN/2 employs a Request/Grant fashion which is essentially centralized. This fundamental difference in their operating methodology of the two protocols is used to analyze the performance of network with RoF employing IEEE 802.11 and HiperLAN/2 in Section III.

\section{A. IEEE 802.11 fundamentals}

The MAC layer of the IEEE 802.11 standard operates in two access mechanisms: Distributed Coordination Function (DCF) and Point Co-ordination Function (PCF). The PCF provides contention-free access, while the DCF adopts the CSMA/CA mechanism for contention-based access. The basic access mechanism, DCF, is illustrated in Figure 2. A station that has a packet ready to send must first "sense" the medium. If the medium is idle, it then waits for an interval of DIFS $\mu$ s (DCFinterframe space) and samples the channel again. If the channel is still idle, it transmits the data packet. Otherwise, it defers the transmission and starts the back-off procedure. At the other end, the receiving station calculates the checksum and determines whether the packet was received correctly. Upon receipt of a correct packet, it waits for SIFS (Short Interframe Space) which is represented as $\mu \mathrm{s}$, and then transmits a positive acknowledgement (ACK) back to the sender [3].

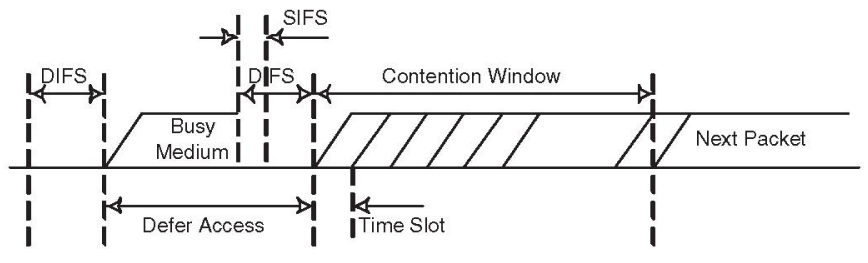

Fig. 2. 802.11 Basic Medium Access Protocol

IEEE 802.11 uses the notion of time slot to define IFS intervals and to calculate the backoff time. Unlike slotted ALOHA, this Slot_Time is not equal to the transmission time of one packet. Under DCF, IEEE 802.11 adopts a slotted binary exponential backoff technique. For each retransmission, the backoff time grows as $\left\lfloor 2^{2+i} *\right.$ ranf ()$\rfloor *$ Slot_Time, where $i$ is the number of consecutive times a station attempts to send the packet, $\operatorname{ranf}()$ is a uniform random variable in $(0,1)$ and $\lfloor x\rfloor$ represents the largest integer less than or equal to $x$.

\section{B. HiperLAN/2 fundamentals}

Unlike IEEE 802.11, the HiperLAN/2 Medium Access Control uses a centralized control mechanism in which Access Points (APs) are responsible for assigning radio resources. HiperLAN's MAC frame has a constant length of $2 \mathrm{~ms}$ and is flexibly structured by phases and time-slots. In other words, the number of time-slots assigned for any MS is not static but is dynamically allocated from frame to frame. As shown in Figure 3, a H/2 frame typically consists of five phases, namely the broadcast phase, the downlink phase, the direct-link phase, the uplink phase and the random access phase.

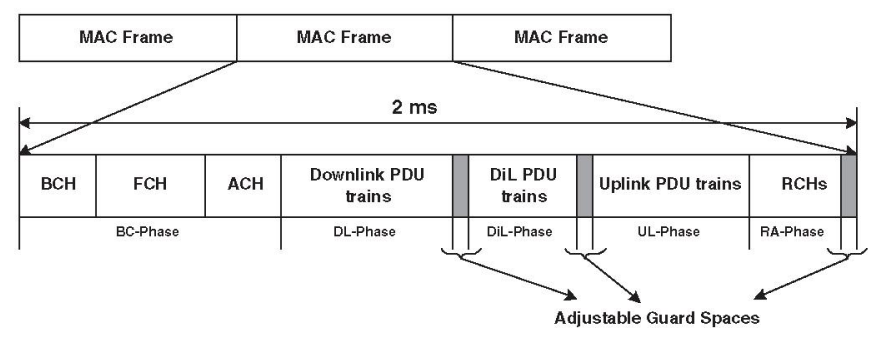

Fig. 3. HiperLAN/2 Frame Structure

The broadcast phase is constituted from three physical channels, i.e. the Broadcast CHannel (BCH), the Frame CHannel $(\mathrm{FCH})$ and the Access Feedback Channel $(\mathrm{ACH})$. Any MS, that wants to access the radio network, initially uses the Random CHannel (in the random access phase) for sending a Resource Request message (RR) to the AP. If a collision occurs during the resource request, the $\mathrm{AP}$ will inform the requesting $\mathrm{MS}$ in the following $\mathrm{ACH}$ and the MS will start a backoff procedure. A RR message contains the number of pending LCHs (Long Transport Channel) in the MS and the AP uses this information for assigning radio resources. 
After a successful RR, which is acknowledged in the ACH by Resource Grant (RG) messages, the MS enters the contention free mode where the MS uses the schedule broadcasted by the AP to send and receive. The schedule sent in the FCH by the AP contains an exact description of the resource allocation within a MAC frame, i.e. the number of time slots during which an MS shall transmit or receive. The length of the $\mathrm{FCH}$ depends on the number of scheduled sessions. The $\mathrm{BCH}$ is used by the AP to broadcast cell information such as the identity of the $\mathrm{AP}$, the transmission power of $\mathrm{AP}$ and the expected received power level at the AP [4].

HiperLAN/2 offers flexible guard time intervals between phases to accommodate different turn-around-time delays. This is a nice feature especially for RoF systems. As specified in the standard, the minimum guard time gap is $2 \mu \mathrm{s}$ which is enough for signals to travel through a $600 \mathrm{~m}$ of multi mode optical fiber. To increase the coverage area of the optical fiber distribution network, we can simply increase this guard time gap. However, as it is analyzed in the next section, this increase in coverage area will be at the cost of network throughput.

\section{MULTIPLE STANDARDS SUPPORT}

In this section, we present an analysis to quantify the effect of the extra propagation delay introduced by the insertion of an optical distribution network into an IEEE 802.11 or a HiperLAN/2 system.

\section{A. IEEE 802.11 employing RoF}

Although the IEEE 802.11 standard specifies the signal's maximum propagation delay to be less than $1 \mu \mathrm{s}$, it is still possible to accommodate the extra propagation delay introduced by the optical distribution network by extending the ACK-timeout value [5]. As long as the timeout is increased to accommodate the ACK packet arriving at the sender before $A C K_{\text {_timeout }}$, the protocol responds properly. Additionally, since IEEE 802.11's DCF specifies that a ready station must wait for DIFS $\mu$ s before it starts the actual transmission, the channel is implicitly reserved for the receiving station to send ACK as long as the ACK arrives during the DIFS interval.

In IEEE 802.11 employing RoF network, the propagation delay due to the signal transmission in the air medium is denoted by $t_{a i r_{-} \text {prog }}$ and the propagation delay caused due to the transmission in the fiber is denoted by $t_{\text {fiber_prog. }}$. We have the following relation: $t_{\text {air }}$ prog $\ll t_{f i b e r_{-} \text {prog }}$. From now on, we denote $t_{\text {prop }}=t_{\text {air_prog }}+t_{\text {fiber_prog }}$ as the total propagation delay of the WLAN network employing RoF.

An underlying assumption in this analysis is that the aggregated traffic generated by all the MSs in a radio cell follows the Poisson distribution with the packet generation rate of $G$ packets $/ \mathrm{s}^{1}$. Hence, $G$ includes new and rescheduled packets. It is also assumed that $t_{\text {prop }}$ is the propagation delay between any pair of MS-RG. This assumption is reasonable since the total propagation delay, $t_{\text {prop }}$, is dominated by the delay introduced due to the transmission in the fiber, $t_{\text {fiber_prog }}$, that

\footnotetext{
${ }^{1}$ In this paper, a radio cell is assumed to span only a room since the whole network operates in a millimeter-wave band
}

is approximately the same for any MS-RG connection. Since during the $t_{\text {prop }}$ duration, it is possible that other stations start their own transmission and thus cause collisions, $t_{\text {prop }}$ is also the vulnerable period of the network ${ }^{2}$. We also assume that all the packets have fixed length and the packet transmission time is constant $L \mu \mathrm{s}$. The transmission of an ACK takes $c \mu \mathrm{s}$.

Further we investigate two network planning scenarios. In the first scenario, each antenna is designed to operate in a separate frequency channel. Similar to cellular network, frequency channels can be reused provided that any two adjacent radio cells operate in different channels. As discussed in Section I, at the millimeter-wave band, each antenna is able to cover only a small confined area such as a room. Consequently, all the MSs connected to the antenna are close to each other and thus are not hidden to each other. However, this scenario is quite unrealistic since the number of MSs inside a room is quite small and the radio resource will not be fully utilized. In the second scenario, a channel is used to serve multiple antennas/rooms. The direct consequence is that MSs in different rooms are separated by walls and hence cannot hear each other. In this case, the MSs in a room are completely hidden to the other MSs in other rooms and thus the RTS/CTS mechanism cannot solve the hidden terminal problem.

1) Scenario 1 - When each antenna operates in a separate radio channel: Let $t$ be the time of arrival of a packet which senses the medium idle. Any other packets arriving in the interval $t$ and $t+t_{\text {prop }}$ will cause a collision. Let $t+Y$ be the time of arrival of the last packet arriving between $t$ and $t+t_{\text {prop }}$. The transmission of any packet arriving in $(t, t+Y)$ will be completed at $t+Y+L$. Only $t_{\text {prop }} \mu$ s later will the channel be sensed idle again. The interval between $t$ and $t+Y+L+t_{\text {prop }}$ is called the transmission period. Using the results from [6], the average duration of $Y$ is given by:

$$
\bar{Y}=t_{\text {prop }}-\frac{1}{G}\left(1-e^{-t_{\text {prop }} G}\right)
$$

The throughput $S_{0}$ of a IEEE 802.11 cell is given by:

$$
S_{0}=\frac{\bar{U}}{\bar{B}+\bar{I}}
$$

where $\bar{U}$ denotes the fragment of the time in a transmission period that the channel is used without collisions, $\bar{B}$ is the expected duration of busy period that is dependent on $Y$ and $\bar{I}$ is the expected duration of the idle period.

The probability that a transmission period is successful is simply the probability that no station transmits during the first $t_{\text {prop }} \mu \mathrm{s}$ of the period, therefore:

$$
\bar{U}=L e^{-t_{p r o p} G}
$$

\footnotetext{
${ }^{2}$ Notice here that we consider the whole period to be vulnerable. In fact, the chance that other stations sending packets and causing collisions is much less likely since both physical and virtual carrier sensing are used in IEEE 802.11
} 
The average duration of an idle period is simply the inversion of the packet generation rate and thus we have

$$
\bar{I}=\frac{1}{G}
$$

The average length of the busy period containing successful transmissions or collisions is given by:

$$
\bar{B}=D I F S+\bar{Y}+L+t_{\text {prop }}+(S I F S+c) e^{-t_{\text {prop }} G}
$$

From Equations (3), (4) and (5), we have the following relation between the throughput of a cell $S_{0}$ and the offered traffic $G$ :

$$
S_{0}=\frac{L e^{-t_{p r o p} G}}{D I F S+\bar{Y}+L+t_{\text {prop }}+(S I F S+c) e^{-t_{p r o p} G}+\frac{1}{G}}
$$

2) Scenario 2 - When multiple antennas operate in the same radio channel: Assume that a radio channel is used to serve $N$ radio cells via an optical fiber distribution network (Fig. 1). An immediate consequence of this configuration is that MSs in one radio cell are completely hidden to MSs in other cells. Hence, it is fair to consider that the $N$ radio cells are independent to each other. As a result, several MSs in different cells can decide that the medium is idle and start sending their packets at the same time. Collision will thus occur at the RG when these signals are collected.

Let $p_{1}$ to be the probability of an arrival of a packet in time $t$ in a cell and $p_{2}$ is the probability of at least one packet arrival in time $t$ in a cell. The probability of only one MS successfully transmits the packet will be: $p_{s}=p_{1}\left(1-p_{2}\right)^{N-1}$. The throughput of the network in this case is:

$$
S_{m}=p_{s} S_{0} .
$$

Under the assumption that the aggregated traffic of the network is Poisson with the packet generation rate of $G$ packets/s, we have $p_{1}=L G e^{-L G}$ and $p_{2}=1-e^{-L G}$.

3) Results: To calculate the throughput $S_{0}$ of a cell, parameters taken from the IEEE $802.11 \mathrm{~b}$ are used. The data rate is $11 \mathrm{Mbps}$ and the SIFS and DIFS intervals are $10 \mu \mathrm{s}$ and $50 \mu$ s successively. Applying the parameters to Equation (6), we obtain the result as shown in Figure 4, that depicts the relation between the throughput $\left(S_{0}\right)$ and the offered traffic in a cell $(G)$ when different propagation delays and packet sizes are used. In this calculation, we assume that $t_{\text {air_prop }}=1 \mu \mathrm{s}$. For the purpose of comparison, throughput of the system is calculated when packet length is 2048 bytes for and fiber lengths of $0 \mathrm{~m}, 1000 \mathrm{~m}, 2000 \mathrm{~m}$ and $4000 \mathrm{~m}$. Moreover, we also consider different packet lengths, i.e. 512, 1024 and 2048 bytes, for the case of $1000 \mathrm{~m}$ of optical fiber.

The result shows that the network throughput does not decrease drastically when extra propagation delay is added. The throughput drops by about $5 \%$ when propagation delay is increased from $1 \mu \mathrm{s}$ ( $0 \mathrm{~m}$ of fiber) to $5 \mu \mathrm{s}$ ( $1000 \mathrm{~m}$ of fiber). Moreover, the network gets saturated more quickly as the fiber length increases. As can also be seen in Figure 4, the throughput of the IEEE 802.11 employing RoF system is degraded drastically when the packet sizes are small. For the

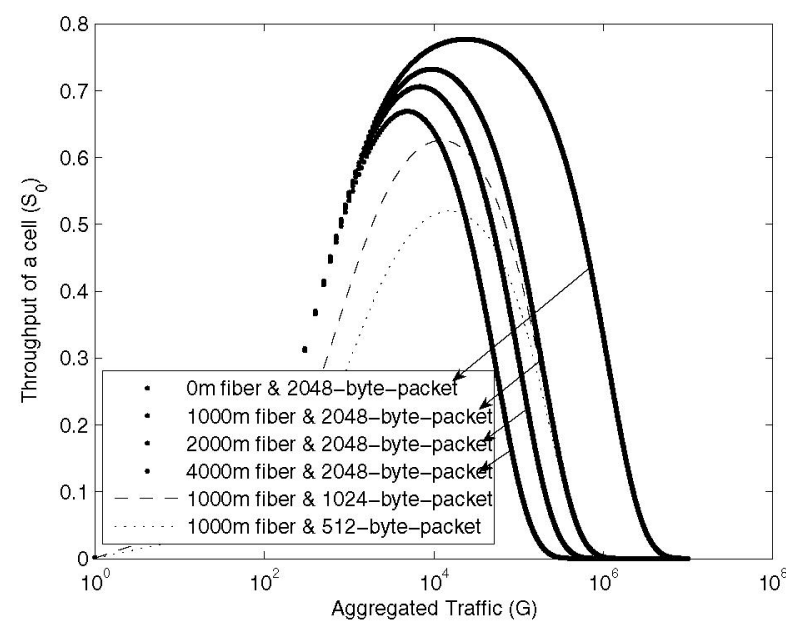

Fig. 4. Approximate throughput of IEEE 802.11 employing Radio over Fiber

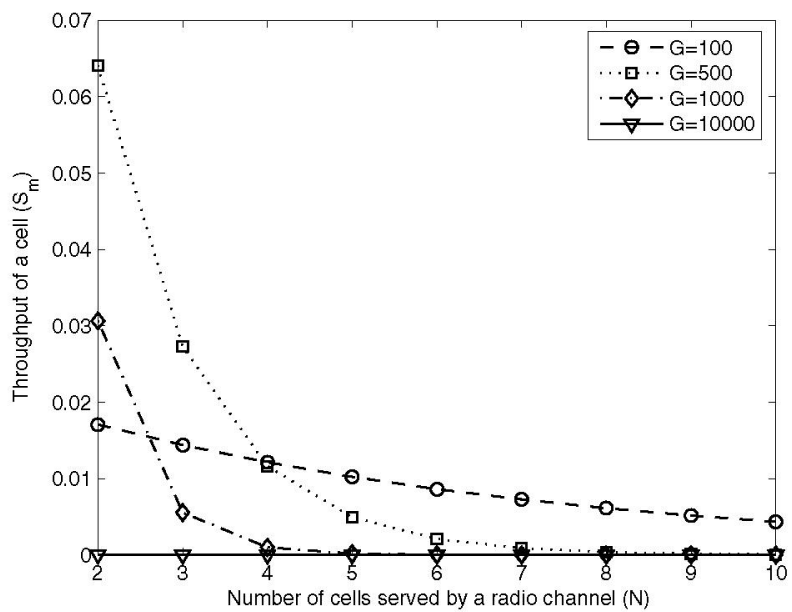

Fig. 5. Throughput of the network when multiple cells are served by an AP with 2048-Byte-packets

case where the packet length is 512 bytes, the maximum throughput is merely above $50 \%$. However, as the packet length is increased, the throughput also raises up to be around $73 \%$ for the case of 2048 bytes packet.

Figure 5 shows the throughput of a cell $\left(S_{m}\right)$ for the second scenario. The number of radio cells $(N)$ served by a single radio channel varies from 2 to 10 . Moreover, different values of the offered traffic in a cell $(G)$ are also used. It is interesting to see that the throughput of a cell in this scenario is unacceptably low. For the case when two radio cells $(N=2)$ are served by one channel and the offered traffic $(G)$ is 500 packets/s, the throughput is merely $6 \%$. As the number of radio cells $(N)$ served by a channel increases, the throughput continues to decrease. Furthermore, when $G$ is 10000 packets/s, indicating that the channel is becoming saturated, the throughput is almost to zero. This phenomenon can be reasoned by the fact that the probability of at least one 
packet arriving in a small duration, say, $t\left(p_{2}=1-e^{-L G}\right)$ goes to 1 when $G$ increases. As a result, the probability of a successful transmission $p_{s}=p_{1}\left(1-p_{2}\right)^{N-1}$ is thus very small. In other words, the probability that two or more MSs in different radio cells/rooms decide to transmit a packet at the same time is close to 1 when the network is saturated. Consequently, the chance that a packet transmission is failed due to collision is also very high in a crowded network. When $G$ is small, indicating an under-served network, the probability of two MSs transmitting at the same time is lower. However the throughput of a cell in the case where there is no hidden terminals $\left(S_{0}\right)$ is also low indicating that the channel is poorly utilized. Consequently, the throughput of a cell $\left(S_{m}\right)$ is very low. This also explains why the throughput $\left(S_{m}\right)$ of a cell when $G=500$ is larger than that when $G=100$.

\section{B. HiperLan/2}

Based on the analysis presented in [7], the effect of the additional propagation delay on the throughput of a HiperLAN/2 network employing RoF is quantified in this section. The length of the LCHs (Long CHannels) that carry the actual data packets is first calculated. The ratio between the LCHs' length (expressed by the number of OFDM symbols) and the total length of a MAC Frame (MF) specifies the maximum throughput of the HiperLAN/2's MAC Protocol. The maximum throughput $\left(T P_{M A X}\right)$ is given by:

$$
T P_{M A X}=N P D U_{L C H} \cdot \frac{x}{\left\lceil\frac{x+4}{48}\right\rceil} \cdot \frac{8}{t_{\text {frame }}}
$$

where $N P D U_{L C H}$ represents the number of LCH PDUs per a MAC frame, $x$ is the length of a user data packet in bytes and $t_{\text {frame }}$ the MAC frame length. $N P D U_{L C H}$ is calculated by:

$$
N P D U_{L C H}=\left\lfloor\frac{L_{L C H} \cdot B p S_{L C H}}{54}\right\rfloor
$$

where $B p S_{L C H}$ is the number of bytes coded in a single OFDM symbol and $L_{L C H}$ is the total length of all the LCHs for the DL, DiL and UL PDU trains in a MAC frame.

To evaluate the network's throughput degradation due to the additional propagation delay introduced by the optical distribution network, different values of the guard time spacing $\left(P_{g}\right)$ are applied to Equation $8^{3}$. For the purpose of simplicity, in this analysis, omnidirectional antennas are used. It is also assumed that each Mobile Terminal has two active Data Link Connections (DLCC) - one is for the uplink and another for the downlink. Moreover, it is further assumed that each active connection has one Short CHannel $(\mathrm{SCH})$ which is used for conveying user control data. Hence, the total number of Information Element (IE) blocks $\left(N_{I E}\right)$ will be double the number of active MTs $\left(N X_{M T}\right)$ in a cell. To plot the effects of increasing guard time spaces accounting for the extra propagation delays $\left(P_{g}\right)$, we assume that there are 10 active Mobile Terminals and $10 \mathrm{RCH}$ slots in a cell. Throughput

\footnotetext{
${ }^{3}$ For the detailed discussion, we refer to [7].
}

is calculated in two cases, i.e. one does not support the DiL phase and the other supports the DiL with 3 pairs of direct link MSs. The result of the case where the DiL phase is not supported is shown in Figure 6. The guard time spacing is chosen to be 2, 4 and $6 \mu$ s that corresponds to $400 \mathrm{~m}, 800 \mathrm{~m}$ and $1200 \mathrm{~m}$ of optical fiber respectively. As can be seen in both Figures 6, the throughput is decreased by about $1 \mathrm{Mbps}$ for each $2 \mu$ s increase in the guard time spacing. This decrease is comparable to the case of a IEEE 802.11 network employing RoF with no hidden nodes.

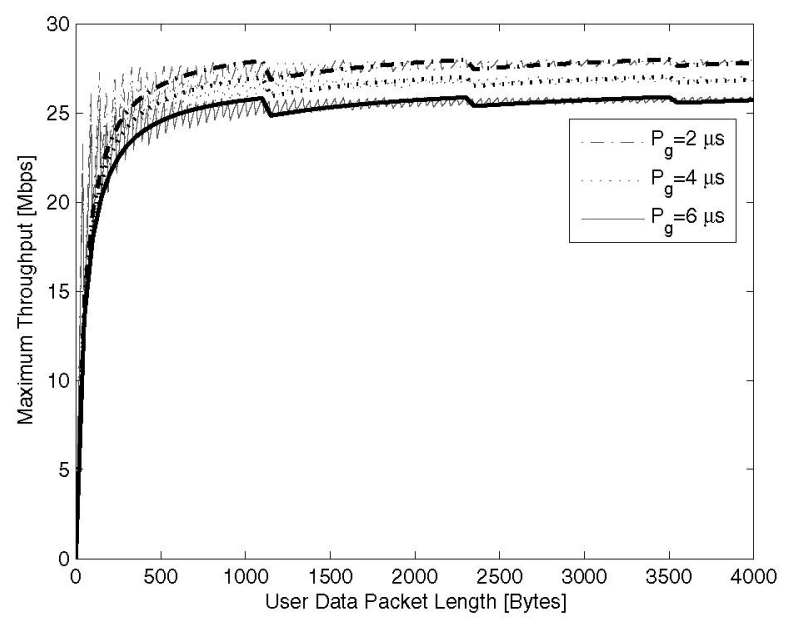

Fig. 6. Throughput by different guard time values - $10 \mathrm{MS}, 10 \mathrm{RCH}$ slots, 0 DiL

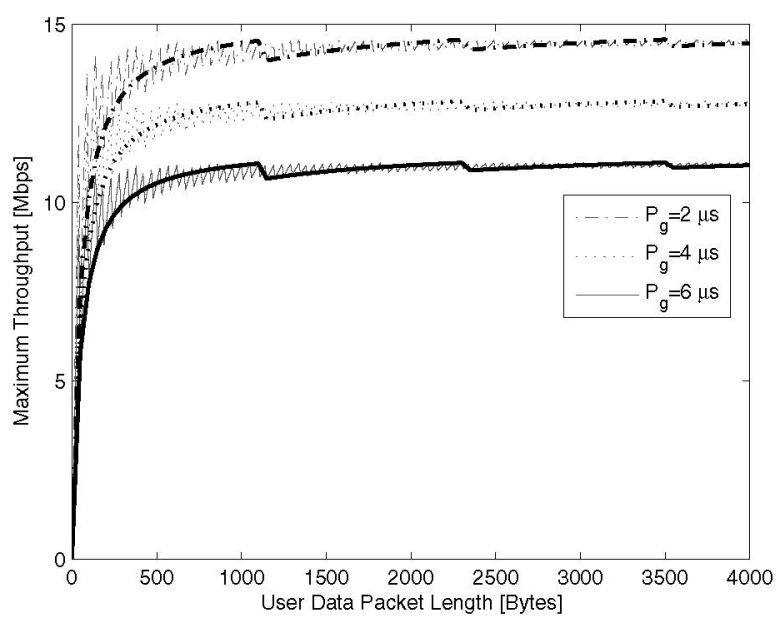

Fig. 7. Throughput by different guard time values including directly link communication - $20 \mathrm{MS}, 10 \mathrm{RCH}$ slots, $3 \mathrm{DiL}$

The results for the second case is shown in Figure 7. Moreover, in this experiment, the number of MSs is increased to 20. As expected, the throughput of the network is much lower than in the first case. This is because of the increase in overhead traffic ( $L_{P R E+S O H}$ [7]) when the number of 
MSs increases. It is also interesting to see that the effect of additional propagation delay is more noticeable in this case as the total length of the guard time spacing in a frame ( $L_{G T S}$ [7]) directly depends on the number of direct link (NDiL) and the number of uplink connections (NUL).

Although HiperLAN/2 is a centralized control protocol, MSs still have to use the Random Access (RA) phase to transmit Resource Request (RR) messages to the RG [8]. As a result, Hiperlan/2 networks employing RoF also suffer from the hidden-terminal problem. However, once an MS has successfully reserved the resource, it enters the contentionfree phase in which resource is explicitly reserved for the MS. As a result, the hidden-terminal problem does not affect the contention-free phases in HiperLAN/2 MAC frames. Moreover, since the number of active connections in a radio cell/room in the indoor environment is normally small, in a HiperLAN/2 network, the probability of two or more RRs packets transmitted at the same time is much less than the probability of collision caused by packets transmission in an IEEE 802.11 network.

\section{CONClusions}

We have described a possible solution for the deployment of future indoor communication network using Radio over Fiber techniques. This architecture is potential to support communications at millimeter wave bands, e.g., $17 \mathrm{GHz}$ or $60 \mathrm{GHz}$. We have also shown that we can directly adopt the existing MAC standards to RoF based networks. We have investigated the effect of the extra propagation delay introduced when an optical distribution system is inserted into the traditional wireless networks. We have shown that both protocols still function correctly when the signal propagation delay is increased. Although the throughput of RoF networks decreases when the propagation delay increases, this decrease is not significant. We have also investigated the problem of hidden terminals in indoor RoF networks at millimeter wave band. The results show that performance of IEEE 802.11 networks at millimeter wave bands is severely degraded and thus is unacceptable. We discussed that for centralized schedule protocols, such as HiperLAN/2, this hidden terminal effect is less severe and thus centralized schedule protocols are favorable for RoF networks. The next step is to confirm these results through simulation studies. Further it is planned to study the effect of mobility of the mobile stations when these two MAC protocols are employed.

\section{ACKNOWLEDGEMENT}

This research was carried out in the Broadband In-home Networks employing Radio over Fiber project within IOP GenCom program funded by the Dutch Ministry of Economics Affairs.

\section{REFERENCES}

[1] P. Smulders, "Exploiting the $60 \mathrm{GHz}$ band for Local Wireless Multimedia Access: Prospects and Future Directions," IEEE Communications Magazine, 2002.

[2] P.P. Smyth, "Optical Radio - A review of a radical new technology for wireless access infrastructure," BT Technology Joumal, vol. 21, no. 3, pp. 22-31, 2003.

[3] ANSI/IEEE Std 802.11, 1999: Wireless LAN Medium Access Control (MAC) and Physical Layer (PHY) Specification, IEEE Std. 802.11, 1999.

[4] Broadband Radio Access Networks (BRAN); HIPERLAN Type 2, ETSI Std., 1999.

[5] Bao Linh Dang, Ignas Niemegeers, "Analysis of IEEE 802.11 in Radio over Fiber Home Networks," 30th IEEE Conference on Local Computer Networks, 2005.

[6] L. Kleinrock and F. A. Tobagi, "Packet Switching in Radio Channels: Part 1-Carrier Sense Multiple-Access Modes and Their Throughput-Delay charateristics," IEEE Transactions on Communications, vol. COM-23, no. 12, Dec 1975.

[7] You-Chang Ko et.al., "Throughput Analysis of ETSI BRAN HIPERLAN/2 MAC Protocol Taking Guard Timing Spaces into Consideration," in Personal Wireless Communications 2004, 2004.

[8] Enzo Mingozzi, "QoS Support by the HiperLAN/2 MAC protocol: A Performance Evaluation," Cluster Computing, no. 5, pp. 145-155, 2002. 